\title{
Stress, inflammation and endometriosis: are patients stuck between a rock and a hard place?
}

\author{
Bettina Toth
}

Published online: 20 February 2010

(C) Springer-Verlag 2010

Endometriosis belongs to the most frequent non-malignant diseases in women during childbearing years [1]. The disease depends on a complex interaction of immunologic, genetic, hormonal and environmental factors often leading to pelvic pain with dysmenorrhoea, dyspareunia and dyschezia $[2,3]$. In the peritoneal cavity of affected women, chemoattraction of macrophages accompanied by immune cell infiltration could be detected [4]. The symptoms of endometriosis are also a product of the local inflammatory response, e.g. tumour necrosis factor alpha and glycodelin correlated positively with the level of menstrual pain in the peritoneal fluid of affected patients [5]. Endometriosis is an oestrogen-dependent disease and established treatment strategies (temporarily) suppress ovarian oestrogen production with known side effects like perimenopausal symptoms. A myriad of possible points of application for progestogens have been postulated. Beside reduced serum oestrogen levels leading to suppressed gonadotropin release, progestogens cause decidualization in eutopic and ectopic endometrium, inhibit angiogenesis by suppressing plasminogen activator activity and decrease intraperitoneal inflammation [6].

Endometriosis is also determined by neurovegetative factors. Pain is a major cause of physical, psycho-social,

B. Toth $(\bowtie)$

Department of Gynecological Endocrinology and Reproductive

Medicine, Ruprecht Karl-University Heidelberg,

Voßstr. 9,

69115 Heidelberg, Germany

e-mail: bettina.toth@med.uni-heidelberg.de emotional and professional or work-related impairment among women with endometriosis. Recently, several studies indicated specific nerve fibres present in endometriotic tissue with existing parallels between density of small nerve fibres and pain severity [6-8]. In a double-blind study, Fraser et al. employed diagnostic laparoscopy and subsequent nerve fibre analysis in endometrial biopsies for diagnostic purposes. They were able to show that nerve fibre analysis in endometrial biopsies was nearly as reliable for diagnosing endometriosis as laparoscopy performed by experienced gynaecological laparoscopists [8]. Fraser et al. were also able to show that combined oral contraceptives and progestogens significantly reduced nerve fibre density and nerve growth factor and cognate receptors in peritoneal endometriotic lesions [6].

Given the severe clinical symptoms of endometriosis, such as chronic pain and infertility, it is not surprising that patients with endometriosis often report poor quality of life, high stress perception and depressive symptoms. Also, patients suffering from endometriosis-related dyspareunia forbear from informing their (sexual) partners implicating a tendency towards a withdrawal and possibly leading to stigmatization and self-pity, further increasing (emotional) stress [9]. Siedentopf et al. [10] reported a reduced quality of life, increased stress perception and depressive symptoms in patients with endometriosis, associated with a peritoneal cytokine profile in favour of inflammation. However, analyses of the ex vivo samples did not allow to confirm a positive correlation between psycho-social and inflammatory markers.

The wealth of published evidence supporting that (1) endometriosis is associated with a poor quality of life and 
high stress perception; (2) endometriosis is an inflammatory disease, and (3) stress and poor quality of life may cause inflammation, strongly suggested that women with endometriosis are stuck between a rock and a hard place within the vicious circle of high stress perception, inflammation and disease progression. The recent study by Tariverdian, Siedentopf, et al. targeted this complex interaction and unveiled a neuroendocrine circuitry in endometriosis, affecting the equilibrium of the endocrine and immune system [11]. They were able to demonstrate that changes of the hormonal axis (low progesterone levels) results in increased inflammation (cytokine secretion by peritoneal cells). Further, they confirmed that mediators of the stress response, such as corticotrophin releasing hormone, aggravated the inflammatory response in peritoneal lymphocytes. Strikingly, this stress hormone induced response could be abrogated in the presence of a progesterone derivative.

One of the pathomechanisms of endometriosis postulates that retrograde menstruation leads to viable endometrial cells in the peritoneal cavity, endometrialperitoneal adhesion and ectopic implantation. An inflammatory environment additionally supports the invasion and maintains endometriosis (Fig. 1). Arck et al. hypothesized that the perpetuation of inflammation results from stress-induced decrease of progesterone opposed by high levels of stress hormones. Hence, disruption of this neuroendocrine circuitry by using progestogens or improved stress coping may be beneficial for patients with endometriosis.

Future research in endometriosis should integrate this "Brain-body-brain cross talk" rather than analysing single pathways. These timely endeavours may help to establish a "fingerprint" for endometriosis and implicate new treatment strategies. Furthermore, stress factors leading to physical, psycho-social, professional or work related and emotional impairment among women with endometriosis should attract notice and coping mechanisms should be established.

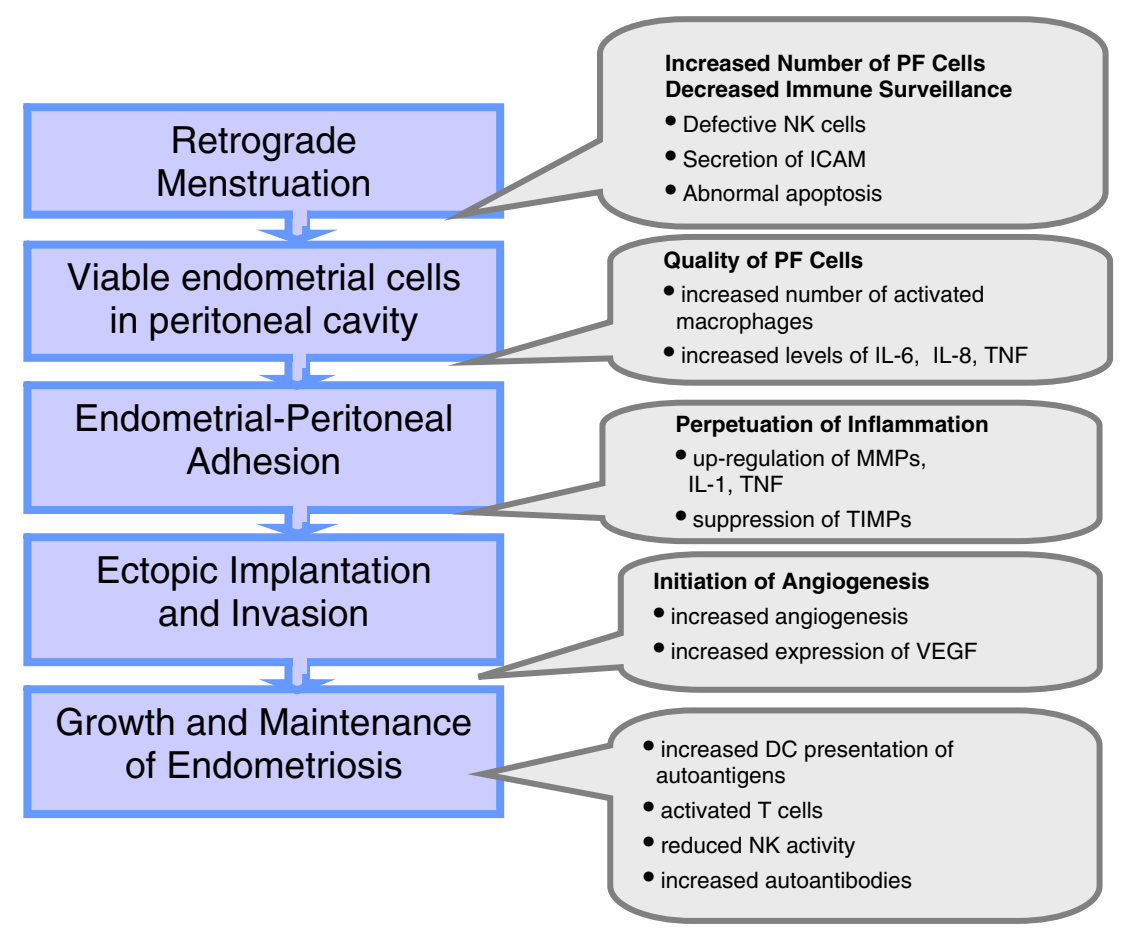

Fig. 1 Pathomechanism of Endometriosis. Retrograde menstruation could lead to viable endometrial cells in the peritoneal cavity with an increased number of cells in the peritoneal fluid $(P F)$ and decreased immune surveillance. The quality of PF cells is dominated by increased numbers of activated macrophages and increased levels of Interleukin (IL)-6, -8 as well as tumour necrosis factor (TNF). Endometrial cells can adhere to the peritoneum further aggravating the perpetuation of inflammation with an up-regulation of MMPs, IL1 and TNF paralleled by suppression of TIMPs. When ectopic implantation and invasion occurs, angiogenesis is mandatory with increased expression of vascular endothelial growth factor $(V E G F)$. Disease progression with growth and maintenance of endometriosis is accompanied by increased dendtritic cell presentation of auto antigens, activated $\mathrm{T}$ cells, reduced natural killer $(N K)$ cell activity and increased autoantibodies. ICAM inter-cellular adhesion molecule, $M M P$ matrix metalloprotease, TIMP tissue inhibitor of metalloprateases. Modified after Kyama et al., Reprod Biol Endocrinol, 2003 [12] 


\section{References}

1. Toth B, Hornung D, Scholz C, Djalali S, Friese K, Jeschke U (2007) Peroxisome proliferator-activated receptors: new players in the field of reproduction. Am J Reprod Immunol 58:289-310

2. Halme J, Becker S, Hammond MG, Raj MH, Raj S (1983) Increased activation of pelvic macrophages in infertile women with mild endometriosis. Am J Obstet Gynecol 145:333-337

3. Klein NA, Pergola GM, Rao-Tekmal R, Dey TD, Schenken RS (1993) Enhanced expression of resident leukocyte interferon gamma mRNA in endometriosis. Am J Reprod Immunol 30:74-81

4. Cirkel U, Ochs H, Mues B, Zwadlo G, Sorg C, Schneider HP (1993) Inflammatory reaction in endometriotic tissue: an immunohistochemical study. Eur J Obstet Gynecol Reprod Biol 48:43-50

5. Scholl B, Bersinger NA, Kuhn A, Mueller MD (2009) Correlation between symptoms of pain and peritoneal fluid inflammatory cytokine concentrations in endometriosis. Gynecol Endocrinol 23:1-6

6. Tokushige N, Markham R, Russell P, Fraser IS (2009) Effect of progestogens and combined oral contraceptives on nerve fibers in peritoneal endometriosis. Fertil Steril 92:1234-1239
7. Bokor A, Kyama CM, Vercruysse L, Fassbender A, Gevaert O, Vodolazkaia A et al (2009) Density of small diameter sensory nerve fibres in endometrium: a semi-invasive diagnostic test for minimal to mild endometriosis. Hum Reprod 24:3025-3032

8. Al-Jefout M, Dezarnaulds G, Cooper M, Tokushige N, Luscombe GM, Markham R et al (2009) Diagnosis of endometriosis by detection of nerve fibres in an endometrial biopsy: a double blind study. Hum Reprod 24:3019-3024

9. Oehmke F, Weyand J, Hackethal A, Konrad L, Omwandho C, Tinneberg HR (2009) Impact of endometriosis on quality of life: a pilot study. Gynecol Endocrinol 23:1-4

10. Siedentopf F, Tariverdian N, Rucke M, Kentenich H, Arck PC (2008) Immune status, psychosocial distress and reduced quality of life in infertile patients with endometriosis. Am J Reprod Immunol 60:449-461

11. Tariverdian N, Rucke M, Szekeres-Bartho J, Blois SM, Karpf EF, Sedlmayr P, et al. (2010) Neuroendocrine circuitry and endometriosis: progesterone derivative dampens corticotropin-releasing hormone-induced inflammation by peritoneal cells in vitro. J Mol Med 10.1007/s00109-009-0559-8

12. Kyama CM, Debrock S, Mwenda JM, D’Hooghe TM (2003) Potential involvement of the immune system in the development of endometriosis. Reprod Biol Endocrinol 2(1):123 